University of Nebraska - Lincoln

DigitalCommons@University of Nebraska - Lincoln

Faculty Publications: Materials Research

Science and Engineering Center

Materials Research Science and Engineering

Center

6-2007

\title{
Effective Demagnetizing Factors of Complicated Particle Mixtures
}

\author{
Ralph Skomski \\ University of Nebraska at Lincoln, rskomski2@unl.edu \\ George C. Hadjipanayis \\ University of Nebraska-Lincoln, hadji@udel.edu \\ David J. Sellmyer \\ University of Nebraska-Lincoln, dsellmyer@unl.edu
}

Follow this and additional works at: https://digitalcommons.unl.edu/mrsecfacpubs

Part of the Materials Science and Engineering Commons

Skomski, Ralph; Hadjipanayis, George C.; and Sellmyer, David J., "Effective Demagnetizing Factors of Complicated Particle Mixtures" (2007). Faculty Publications: Materials Research Science and Engineering Center. 71.

https://digitalcommons.unl.edu/mrsecfacpubs/71

This Article is brought to you for free and open access by the Materials Research Science and Engineering Center at DigitalCommons@University of Nebraska - Lincoln. It has been accepted for inclusion in Faculty Publications: Materials Research Science and Engineering Center by an authorized administrator of DigitalCommons@University of Nebraska - Lincoln. 


\title{
Effective Demagnetizing Factors of Complicated Particle Mixtures
}

\author{
Ralph Skomski ${ }^{1}$, G. C. Hadjipanayis ${ }^{2}$, and D. J. Sellmyer ${ }^{1}$ \\ ${ }^{1}$ Department of Physics and Astronomy and the Nebraska Center for Materials and Nanoscience, University of Nebraska, \\ Lincoln, NE 68588 USA \\ ${ }^{2}$ Department of Physics and Astronomy, University of Delaware, Newark, DE 19716 USA
}

\begin{abstract}
Demagnetizing factors have been calculated for hierarchical mixtures and dispersions of magnetic particles, such as columns of platelike particles in a matrix and clusters of spherical particles. The theory involves a number of shape and density parameters describing particles, aggregates, and matrix. It approximates distant particles by a homogeneous medium and yields closed and easy-to-use expressions for the effective demagnetizing factor.
\end{abstract}

Index Terms-Biomagnetism, demagnetizing fields, magnetic particles.

\section{INTRODUCTION}

$\mathbf{I}$ $\mathrm{N}$ contrast to isolated ellipsoids [1], the definition and determination of demagnetizing factors $D$ for general ferromagnets has remained a complicated question. This refers not only to the values of the demagnetizing factors but also to the applicability of the concept of demagnetizing fields [2]. For homogeneously dispersed particles (Fig. 1), there exist simple approximate formulae [3], but the particle positions are often correlated due to magnetic or rheological interactions. This includes particles in a nonmagnetic solid or liquid matrix, such as bonded permanent magnets and magnetic nanoparticles in blood [4]. In the latter case, the demagnetizing field is of direct importance, because it affects the transport of magnetic particles used for targeted drug administration. Two exemplary experimental systems are powders of elongated nanoparticles particles, such as $\mathrm{Fe}$, and alnico-type permanent magnets, where long needles of $\mathrm{Fe}_{65} \mathrm{Co}_{35}$ are embedded in a nonmagnetic matrix Al-Ni matrix.

In this paper, we consider the demagnetizing field in aggregates of particles embedded in a matrix. We start with a brief summary of demagnetizing fields in ellipsoids of revolution, derive demagnetizing factors for hierarchical mixtures and dispersions, and finally discuss the merits and shortcomings of the demagnetizing-field approach.

\section{MicRomagnetic BACKGROUND}

The demagnetizing field is an approach to treat the magnetostatic selfinteraction $-1 / 2 \mu_{\mathrm{o}} \int \mathbf{M} \cdot \mathbf{H}_{\mathrm{d}} \mathrm{d} V$, where $\mathbf{H}_{\mathrm{d}}$ is a vector functional (integral function) of $\mathbf{M}(\mathbf{r})$. In ellipsoids of revolution that are homogeneously magnetized along the axis of symmetry, the internal self-interaction field is equal to the demagnetizing field, $\mathbf{H}_{\mathrm{d}}=-D \mathbf{M}$. Throughout Secitons II and III we assume that this homogeneity condition is satisfied.

The demagnetizing field may be calculated directly, by field integration, but it is often convenient to start from Maxwell's equations. First, in the absence of macroscopic currents, $\nabla \times$ $\mathbf{H}=0$ and the field component $H_{\|}$parallel to any surface is continuous. For needle-shaped ellipsoids, this means that $H_{\mathrm{D}}=$ 0 and $D=0$. Second, $\nabla \cdot \mathbf{B}=0$ means that the normal

Digital Object Identifier 10.1109/TMAG.2007.893798

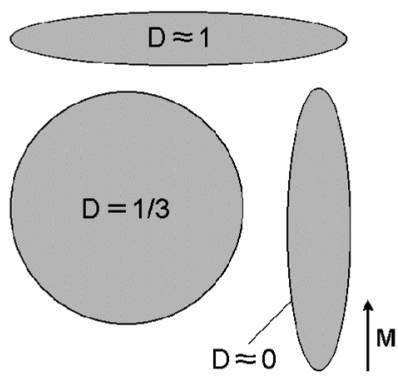

(a)

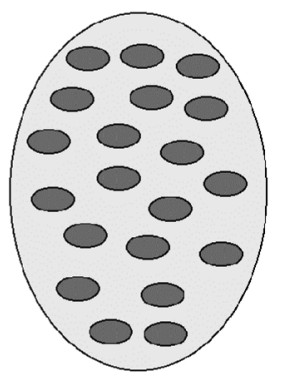

(b)
Fig. 1. Isolated and embedded particles: (a) isolated ellipsoids and (b) simple mixtures.

flux-density component $B_{\perp}$ is continuous at any surface. For strongly oblate or plate-like magnets, this leads to $H_{\mathrm{D}}=-M$ and $D=1$.

Demagnetizing factors for general ellipsoids were discussed by Osborn [1]. For prolate and oblate ellipsoids of revolution with intermediate aspect ratios $\kappa_{\mathrm{o}}=R_{\mathrm{z}} / R_{\mathrm{x}}$ [3]

$$
D=\frac{1}{\kappa_{\mathrm{o}}^{2}-1}\left(\frac{\kappa_{\mathrm{o}}}{\sqrt{\kappa_{\mathrm{o}}^{2}-1}} \operatorname{arcosh} \kappa_{\mathrm{o}}-1\right)
$$

and

$$
D=\frac{1}{1-\kappa_{\mathrm{o}}^{2}}\left(1-\frac{\kappa_{\mathrm{o}}}{\sqrt{1-\kappa_{\mathrm{o}}^{2}}} \arccos \kappa_{\mathrm{o}}\right)
$$

respectively ( $R_{\mathrm{x}}$ and $R_{\mathrm{z}}$ are the equatorial and polar radii, respectively). As shown in Fig. 1(a), spherical particles exhibit $D=1 / 3$. In the limits of needle-shaped $\left(\kappa_{\mathrm{o}} \gg 1\right)$ and platelike $\left(\kappa_{\mathrm{O}} \ll 1\right)$ magnets, the respective expressions reduce to $D=\left(\ln \kappa_{o}-0.307\right) / \kappa_{\mathrm{o}}^{2}$ and $D=1-\pi \kappa_{\mathrm{o}} / 2$. Two example are 0.527 and 0.174 for aspect ratios of 0.5 and 2.0, respectively. Equation (1) is the starting point for the following considerations.

\section{MODEl AND CALCUlATION}

Fig. 2 shows the basic geometry of the investigated magnetic systems. The macroscopic magnets, characterized by a global demagnetizing factor $D_{\mathrm{g}}$, contain aggregates of magnetic particles. The particles and aggregates may have shapes 


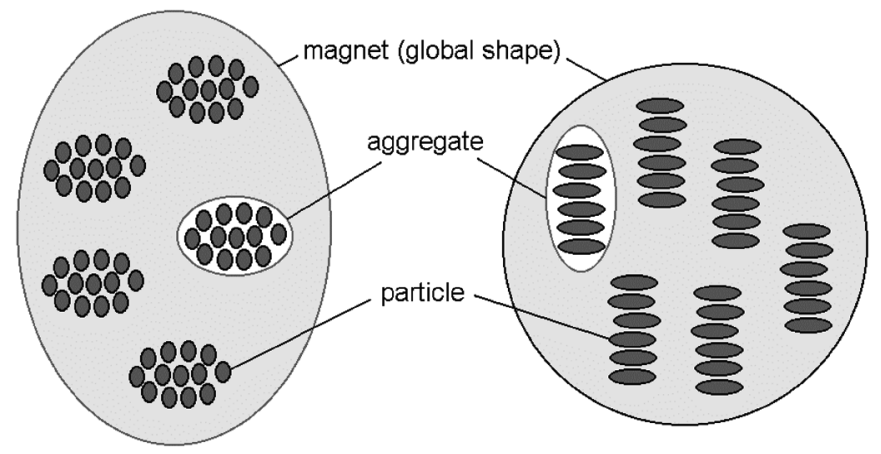

Fig. 2. Hierarchical mixtures: (a) elongated ellipsoids in platelets (left) and (b) columnar aggregates of platelets.

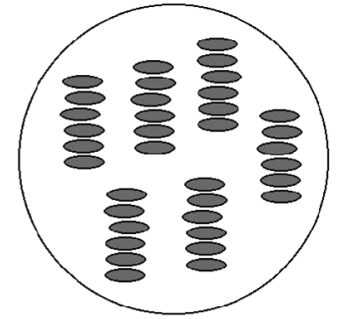

(a)

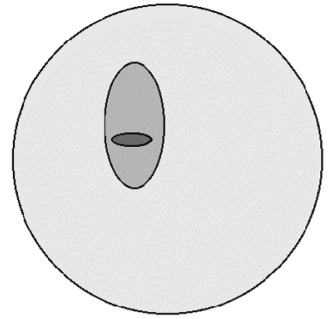

(b)
Fig. 3. Demagnetizing field approximation for a complicated mixture: (a) real structure and (b) effective-medium approximation.

different from the global shape and are characterized by demagnetizing factors $D_{\mathrm{p}}$ and $D_{\mathrm{a}}$ respectively. Examples are chains of plate-like $\left(D_{\mathrm{p}} \approx 1\right)$ or spherical $\left(D_{\mathrm{p}} \approx 1 / 3\right)$ particles embedded in a thin film $\left(D_{\mathrm{a}}=0\right.$ and $\left.D_{\mathrm{g}}=1\right)$ and elongated clusters of parallel magnetic needles in a bulk magnet $\left(D_{\mathrm{p}}=0, D_{\mathrm{a}}<1 / 3, D_{\mathrm{g}} \approx 1 / 3\right)$. The volume fraction of the aggregates in the global matrix is $f_{\mathrm{a}}$, whereas the volume fraction of the particles in the aggregates is $f_{\mathrm{p}}$. This yields the aggregate magnetization $f_{\mathrm{p}} M_{\mathrm{p}}$ and the global magnetization $f_{\mathrm{a}} f_{\mathrm{p}} M_{\mathrm{p}}$, where $M_{\mathrm{p}}$ is the magnetization of the particles.

In this paper, we calculate the demagnetizing field as the sum over all interparticle interaction fields plus the particles' own demagnetizing field. To perform the calculation, we approximate distant particles by a homogenously magnetized medium and exploit that magnetic fields in homogeneously magnetized ellipsoids of revolution are homogeneous. Fig. 3 shows how a complicated mixture [Fig. 3(a)] is approximated by a hierarchy of embedded ellipsoids [Fig. 3(b)]. Since the field inside a homogeneously magnetized ellipsoid of revolution is homogeneous, it does not matter whether the embedded ellipsoids are centered or not.

Each surface or interface in Fig. 3(b) adds a demagnetizing field $D_{\mathrm{I} / \mathrm{II}}\left(M_{\mathrm{I}}-M_{\mathrm{II}}\right)$, where $M_{\mathrm{I}}$ and $M_{\mathrm{II}}$ are the magnetizations of the outer and inner regions, respectively. The demagnetizing field is obtained by adding the contributions from the magnet's surface (global contribution), from the aggregates, and

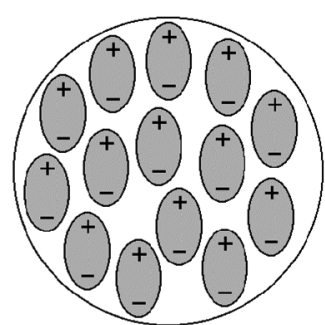

(a)

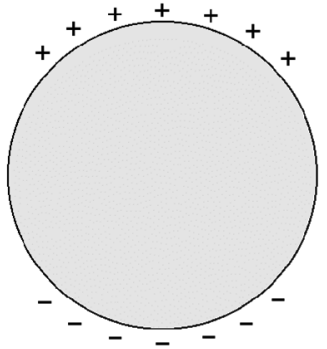

(b)
Fig. 4. Charge distribution in a simple mixture: (a) local charges and (b) net charges at the surface of an aggregate.

from the particle's own surface. This yields the demagnetizing factor

$$
D=D_{\mathrm{p}}\left(1-f_{\mathrm{p}}\right)+D_{\mathrm{a}} f_{\mathrm{p}}\left(1-f_{\mathrm{a}}\right)+D_{\mathrm{g}} f_{\mathrm{a}} f_{\mathrm{p}} .
$$

For $f_{\mathrm{a}}=1, D$ reduces to the expression for the simple mixtures shown in Fig. 1(b)

$$
D=D_{\mathrm{p}}\left(1-f_{\mathrm{p}}\right)+D_{\mathrm{g}} f_{\mathrm{p}} .
$$

This equation interpolates between the particle demagnetizing factor $D_{\mathrm{p}}$ for small particle concentrations $f_{\mathrm{p}}=0$ and the "global" demagnetizing factor $D_{\mathrm{g}}$ for $f_{\mathrm{p}}=1$.

So far, we have restricted ourselves to a nonmagnetic matrix. The generalization to magnetic particles embedded in a magnetic matrix is straightforward and realized by exploiting $D_{\mathrm{I} / \mathrm{II}}\left(M_{\mathrm{I}}-M_{\mathrm{II}}\right)$. For example, (3) now becomes

$$
D=\left(D_{\mathrm{p}}\left(1-f_{\mathrm{p}}\right)+D_{\mathrm{g}} f_{\mathrm{p}}\right)\left(1-\frac{M_{\mathrm{o}}}{M_{\mathrm{p}}}\right)+D_{\mathrm{g}} \frac{M_{\mathrm{o}}}{M_{\mathrm{p}}}
$$

where $M_{\mathrm{o}}$ is the magnetization of the matrix. For $M_{\mathrm{o}}=M_{\mathrm{p}}$, this reduces to $D=D_{\mathrm{g}}$, as expected for a homogeneous system.

\section{DISCUSSION}

The demagnetizing-field approximation is well-adapted to some problems but fails when applied in an improper context. In the fooling subsections, we discuss some aspects of applicability of demagnetizing factors.

\section{A. Local Magnetic Fields}

The quality of the demagnetizing-field approach depends on the fluctuations of the local magnetic field. Homogeneously magnetized bodies of arbitrary shape have their magnetic charges at the surface, but most magnets are structurally inhomogeneous, so that $\nabla \cdot M \neq 0$ inside the magnet. Fig. 4(a) illustrates that positive and negative magnetic charges largely but not completely cancel each other. The approach of Section III corresponds to Fig. 4(b), so that residual fluctuations go beyond (2)-(4).

A striking example of a nonellipsoidal effect is the demagnetizing field in the middle of a long rod, which is much smaller 
than that in an elongated ellipsoid of revolution having the same aspect ratio. This is because ellipsoids have magnetic charges not only at the top and bottom but also (to a lesser extent) at the sides.

An interesting point is the relation between demagnetizing and interaction fields. So long as the spin distribution is fixed or-more generally - uncorrelated, demagnetizing and interaction fields are largely equivalent. This makes it possible to express the micromagnetic energy of an ensemble of small particles in terms of interaction energies.

\section{B. Incoherent Magnetization States}

The demagnetizing field is based on the assumption of a parallel spin orientation throughout the magnet, generally going beyond the $c$-axis alinment assumed in (2)-(4). This limit is rarely realized in practice, because magnetostatic flux closure favors domains and other incoherent magnetization states, accompanied by field corrections comparable to DM. Examples are the transition from coherent rotation (or Stoner-Wohlfarth behavior) to curling in single-domain particles [5], [6] and cooperative magnetization reversal in chains and rings of nanoparticles [6].

The trend towards domain formation competes with the interatomic exchange, which favors parallel spin orientation. Since interatomic exchange is a short-range phenomenon, the shapeanisotropy model works best for small magnetic particles.

\section{Demagnetizing Factors and Shape Anisotropy}

The demagnetizing field $H_{\mathrm{D}}$ caused by the poles on the magnet's surface should not be confused with the shape-anisotropy field $2 K_{\mathrm{sh}} / \mu_{\mathrm{O}} M_{\mathrm{s}}$. Both phenomena involve the demagnetizing factor $D$, but the physics is different. The demagnetizing field is always negative, $H_{\mathrm{D}}=-D M$, whereas the shape-anisotropy field can have either sign. For example, the Stoner-Wohlfarth coercivity

$$
H_{\mathrm{c}}=\frac{2 K_{1}}{\mu_{\mathrm{o}} M_{\mathrm{s}}}+\frac{1}{2}(1-3 D) M_{\mathrm{s}}
$$

contains the shape-anisotropy field $1 / 2(1-3 D) M_{\mathrm{s}}$, which differs from the demagnetizing field $-D M_{\mathrm{s}}$. This indicates that the demagnetizing field is more than just an additional external-field contribution. For example, the demagnetizing field in a sphere ( $D=1 / 3)$ is $-M_{\mathrm{s}} / 3$, as contrasted to the absence of shape anisotropy in spheres.

\section{Relation to Effective-Medium Theories}

Effective fields are different from effective materials parameters, such as effective susceptibilities. The Bruggeman theory [7] yields materials parameters for two-phase composites on a mean-field level. The most general equation for a two-phase mixture is [8]

$$
f \frac{Q_{\mathrm{II}}-Q_{\mathrm{eff}}}{g Q_{\mathrm{II}}+(1-g) Q_{\mathrm{eff}}}+(1-f) \frac{Q_{\mathrm{I}}-Q_{\mathrm{eff}}}{g Q_{\mathrm{I}}+(1-g) Q_{\mathrm{eff}}}=0
$$

where $Q_{\mathrm{I}}$ and $Q_{\mathrm{II}}$ are the materials parameters of two phases, $f$ is the volume fraction of embedded phase II, and $g$ is a system and geometry-dependent parameter. For magnetic susceptibilities $\left(Q_{\text {eff }}=\chi_{\text {eff }}\right)$, the parameter $g$ is equal to the demagnetizing factor $D$ of the second phase, and the solution of (6) is

$$
\begin{aligned}
\chi_{\mathrm{eff}}= & \frac{\chi_{\mathrm{II}}(\phi-D)+\chi_{\mathrm{I}}(1-D-\phi)}{2(1-D)} \\
& \times\left(1 \pm \sqrt{1+\frac{4 \chi_{\mathrm{II}} \chi_{\mathrm{I}} D(1-D)}{\left(\chi_{\mathrm{II}}(\phi-D)+\chi_{\mathrm{I}}(1-D-\phi)\right)^{2}}}\right) .
\end{aligned}
$$

This equation interpolates between $\chi_{\mathrm{I}}(\phi=0)$ and $\chi_{\mathrm{II}}(\phi=$ $1)$. For $\chi_{\mathrm{I}} \approx \chi_{\mathrm{II}}$, it reduces to $\chi_{\mathrm{eff}}=\langle\chi\rangle$, but in general the dependence on $\phi$ is strongly nonlinear.

\section{CONCLUSION}

In summary, we have investigated the demagnetizing-field behavior of complex mixtures. We have derived effective-medium expression for hierarchical mixtures and magnetic particles embedded in magnetic matrix. The demagnetizing factors are a useful tool to describe interaction effects but fail if interactions change the relative spin orientations of the particles.

\section{ACKNOWLEDGMENT}

This work was supported by National Science Foundation (NSF), in part by the Materials Research Science \& Engineering Center (MRSEC), and in part by the Nebraska Center for Materials and Nanoscience (NCMN). The author authors would like to thank D. J. Sellmyer and D. Leslie-Pelecky for helpful discussions.

\section{REFERENCES}

[1] J. A. Osborn, "Demagnetizing factors of the general ellipsoid," Phys. Rev., vol. 67, pp. 351-357, 1945.

[2] R. Skomski, J.-P. Liu, and D. J. Sellmyer, "Quasicoherent nucleation mode in two-phase nanomagnets," Phys. Rev. B, vol. 60, pp. 7359-7365, 1999.

[3] R. Skomski and J. M. D. Coey, "Permanent magnetism," Institute of Physics. Bristol, U.K., 1999.

[4] V. Labhasetwar and D. L. Leslie-Pelecky, Eds., Biomedical Applications of Nanotechnology. New York: Wiley, 2006.

[5] A. Aharoni, Introduction to the Theory of Ferromagnetism. Oxford, U.K.: Oxford Univ. Press, 1996.

[6] R. Skomski, "Nanomagnetics," J. Phys.: Condens. Matter, vol. 15, pp. R841-896, 2003.

[7] D. A. G. Bruggeman, "Berechnung verschiedener physikalischer Konstanten von heterogenen Substanzen," Annalen der Physik, vol. 5, no. 24, pp. 637-679, 1935.

[8] R. Skomski, J.-Y. Li, J. Zhou, and D. J. Sellmyer, M. Chipara, D. L. Edwards, R. S. Benson, and S. Phillips, Eds., "Multiscale phenomena in bruggeman composites," in Proc. Mater. Res. Soc. Symp, 2005, vol. 851, Materials for Space Applications, Paper NN1.7.

Manuscript received October 31, 2006; revised January 30, 2007 (e-mail: rskomski@neb.rr.com). 